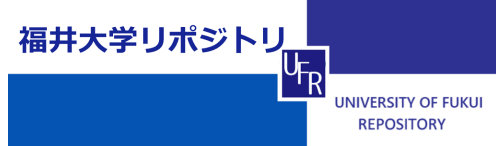

Strongl y subl umi nal regi me of opt i cal - t o- ter ahert z conver si on i n GaP

\begin{tabular}{|l|l|}
\hline 著者 & $\begin{array}{l}\text { BAKUNOV M I ., TSAREV M V., BODROV S. B. , } \\
\text { TAN Nasahi ko }\end{array}$ \\
\hline $\begin{array}{l}\text { j our nal or } \\
\text { publ i cat } \mathrm{i} \text { on t i t l e }\end{array}$ & Jour nal of Appl i ed Physi cs \\
\hline vol une & 105 \\
\hline number & 8 \\
\hline page r ange & $083111-1$ - 083111- 9 \\
\hline year & $2009-04$ \\
\hline URL & ht t p: //hdl . handl e. net /10098/2107 \\
\hline
\end{tabular}




\title{
Strongly subluminal regime of optical-to-terahertz conversion in GaP
}

\author{
M. I. Bakunov, ${ }^{1,2, a)}$ M. V. Tsarev, ${ }^{1,2}$ S. B. Bodrov, ${ }^{1,2}$ and M. Tani ${ }^{3}$ \\ ${ }^{1}$ University of Nizhny Novgorod, Nizhny Novgorod 603950, Russia \\ ${ }^{2}$ Institute of Applied Physics, Russian Academy of Sciences, Nizhny Novgorod 603950, Russia \\ ${ }^{3}$ Research Center for Development of Far-Infrared Region, University of Fukui, Fukui 910-8507, Japan
}

(Received 18 December 2008; accepted 26 February 2009; published online 27 April 2009)

\begin{abstract}
We introduce and investigate a special regime of terahertz generation in electro-optic crystals with ultrashort laser pulses, in which neither phase-matching nor Cherenkov radiation mechanism are efficient. This regime occurs under the conditions that the optical group refractive index is larger than the low-frequency phase refractive index of the crystal, and the spectral bandwidth of the laser pulse is smaller than the frequency of the phase-matched terahertz wave. In this regime, that can be realized for a Ti:sapphire laser in GaP, terahertz generation is provided by transient processes at the crystal boundaries. We study the peculiarities of the terahertz emission from a slab of GaP excited with a Ti:sapphire laser. In particular, we demonstrate a possibility to implement a pointlike source of terahertz radiation that can increase the resolution of terahertz apertureless near-field microscopy. (c) 2009 American Institute of Physics. [DOI: 10.1063/1.3110064]
\end{abstract}

\section{INTRODUCTION}

Optical rectification of ultrashort laser pulses propagating through electro-optic crystals is a widely used technique for generating broadband terahertz radiation. In this technique, the pump optical pulse produces a nonlinear polarization, which follows the intensity envelope of the pulse. The nonlinear polarization moves with the group velocity of the optical pulse and emits terahertz radiation.

From the kinematic point of view, the emission is classified into two regimes depending on the dispersion properties of the electro-optic material-the superluminal, with $n_{g}$ $<n_{0}$, and subluminal, with $n_{g}>n_{0}$, regimes, where $n_{g}$ is the optical group refractive index and $n_{0}$ is the low-frequency phase refractive index of the material. ${ }^{1,2}$

In the superluminal regime $\left(n_{g}<n_{0}\right)$, the optical pulse can produce terahertz radiation via Cherenkov radiation mechanism; the moving nonlinear polarization emits terahertz radiation very much like a relativistic dipole emits Cherenkov radiation. ${ }^{3}$ To produce the Cherenkov cone of terahertz waves the optical pulse should be focused to a size of the order of (or smaller than) the terahertz wavelength. In the subluminal regime $\left(n_{g}>n_{0}\right)$, there is always a frequency $\omega_{0}$ at which phase-matching is achieved, owing to the presence of terahertz dispersion $n_{g}=n\left(\omega_{0}\right)$, where $n(\omega)$ is the phase refractive index in the terahertz range. This provides a simple way to generate terahertz radiation; illumination of a slab of subluminal material by a large (as compared to the terahertz wavelength) aperture beam of femtosecond laser pulses results in the phase-matched excitation of a quasiplane terahertz wave propagating collinearly to the laser beam.

In Ref. 4, it was proposed to distinguish two cases in the superluminal regime of terahertz generation-the strongly and weakly superluminal regimes. In the strongly superluminal regime, the difference between $n_{g}^{2}$ and $n_{0}^{2}$ is significantly

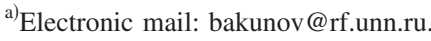

larger than the variation in $n^{2}(\omega)$ over the spectral bandwidth of the laser pulse $\sim 2 \tau^{-1}$ ( $\tau$ is the laser pulse duration), i.e., $n_{0}^{2}-n_{g}^{2} \gg n^{2}\left(2 \tau^{-1}\right)-n_{0}^{2}$, and, therefore, the role of terahertz dispersion in the optical-to-terahertz conversion is negligible. This regime is typical, for example, for $\mathrm{LiNbO}_{3}$ excited with Ti:sapphire laser. ${ }^{4}$ In the weakly superluminal regime when $n_{0}^{2}-n_{g}^{2} \leqslant n^{2}\left(2 \tau^{-1}\right)-n_{0}^{2}$ (as for GaAs excited with a fiber laser ${ }^{4}$ ), the dispersion significantly affects the generated terahertz fields.

In this paper, we go further and show that the subluminal regime of terahertz generation can be usefully classified into two cases as well-the weakly and strongly subluminal regimes according to the conditions $\omega_{0} \tau \lesssim 2$ and $\omega_{0} \tau \gg 2$, respectively. These conditions, unlike the kinematic conditions of the strongly and weakly superluminal regimes, have dynamic nature-they define how efficient the excitation of the phase-matched wave is. Indeed, optical rectification of an ultrashort laser pulse can be considered as a result of difference-frequency mixing among the spectral components within the pulse bandwidth. Apparently, the maximal frequency of the generated terahertz spectrum is restricted by the spectral bandwidth of the pump optical pulse. For $\omega_{0} \tau$ $\lesssim 2$, the bandwidth $2 \tau^{-1}$ is comparable to (or even larger than) the phase-matched frequency $\omega_{0}$ and, therefore, the phase-matched wave is excited efficiently. As a typical example of the weakly subluminal regime, we refer to the widely used technique ${ }^{5-7}$ - phase-matched generation of $\sim 2.5 \mathrm{THz}$ wave in ZnTe by Ti:sapphire laser pulses with typical duration $\tau_{\mathrm{FWHM}} \lesssim 250$ fs, i.e., $\omega_{0} \tau \lesssim 2.3\left[\tau_{\mathrm{FWHM}}\right.$ is the standard full width at half maximum (FWHM) duration for a Gaussian pulse, $\left.\tau_{\mathrm{FWHM}}=2 \sqrt{\ln 2} \tau \approx 1.67 \tau\right]$. In the strongly subluminal regime $\left(\omega_{0} \tau \gg 2\right)$, which is introduced in this paper, phase-matching with a collinearly propagating terahertz wave is allowed kinematically (there exists a phase-matched frequency $\omega_{0}$ ) but the wave is practically not excited. Its amplitude is negligible because the frequency $\omega_{0}$ exceeds the maximal generated frequency $\sim 2 \tau^{-1}$. The strongly subluminal regime can be realized in $\mathrm{GaP}$ excited with a Ti:sapphire 


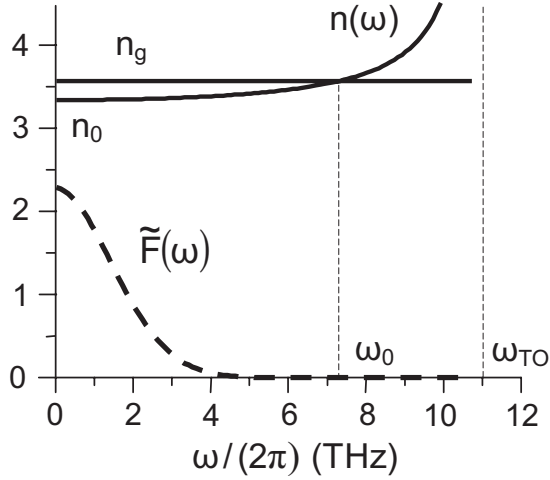

FIG. 1. Illustration of the conditions of the strongly subluminal regime of optical-to-terahertz conversion. The intersection point of $n_{g}$ with $n(\omega)$ defines the phase-matched frequency $\omega_{0}$. The spectrum of the optical intensity envelope $\widetilde{F}(\omega)$ is negligible at $\omega=\omega_{0}$. Parameters are taken for GaP pumped at $800 \mathrm{~nm}$ by a Gaussian pulse with $\tau_{\mathrm{FWHM}}=150$ fs.

laser pulse of a typical duration (Fig. 1). The peculiarity of $\mathrm{GaP}$ is a relatively high frequency of the first transverse optical $(\mathrm{TO})$ phonon resonance $\omega_{\mathrm{TO}} /(2 \pi) \approx 11 \mathrm{THz}$ [compare, for example, with ZnTe where $\omega_{\mathrm{TO}} /(2 \pi) \approx 5.3 \mathrm{THz}$. Due to this fact phase matching between a Ti:sapphire laser pulse and a collinearly propagating terahertz wave is achieved at a rather high frequency $\omega_{0} /(2 \pi) \approx 7.4 \mathrm{THz}$ (Fig. 1). This frequency lies outside the bandwidth of the generated terahertz spectrum for the pump pulse durations $\tau_{\mathrm{FWHM}} \gg 70$ fs $\left(\omega_{0} \tau\right.$ $\gg 2$ ) and, therefore, the phase-matched wave is practically not excited. Additionally, due to the high resonance frequency $\omega_{\mathrm{TO}}$ the dispersion curve in the range of a few terahertz is flat what prevents dispersion broadening of the generated terahertz pulse. As we show further, this enables one to produce single-cycle terahertz pulses.

Recently, GaP has drawn attention as an attractive material for terahertz generation with use of Yb-doped fiber systems operating at $\gtrsim 1.055 \mu \mathrm{m}$ wavelengths. ${ }^{8}$ Since $n_{g}$ in $\mathrm{GaP}$ at $1.055 \mu \mathrm{m}$ is slightly smaller than $n_{0}\left[n_{g}=3.30, n_{0}\right.$ $=3.34$ (Ref. 9)], the generation regime that was exploited in Ref. 8 may be referred to, according to classification of Ref. 4 , as weakly superluminal.

Below we develop a theory of the strongly subluminal regime of terahertz generation in a slab of $\mathrm{GaP}$ excited with a Ti:sapphire laser. We consider a two-dimensional (2D) case when the pump laser pulse is infinite in one direction and has a finite width in the other direction. The 2D case does not require very bulky calculations as the three-dimensional (3D) case of focusing to a spot. At the same time, it allows us to study the effect of laser focusing on the generated terahertz fields. In practice, focusing pump laser pulse into a line by a cylindrical lens offers several advantages,${ }^{4}$ in particular, it is used to scale up the energy of terahertz pulses created by optical rectification. ${ }^{10,11}$

Our consideration is based on the developed, in Ref. 4, general theoretical approach to terahertz generation in a slab of an electro-optic material with focused to a line laser pulses. Applying this approach to GaP excited with a Ti:sapphire laser, we trace the temporal dynamics of the optical-toterahertz conversion inside the slab and study waveforms

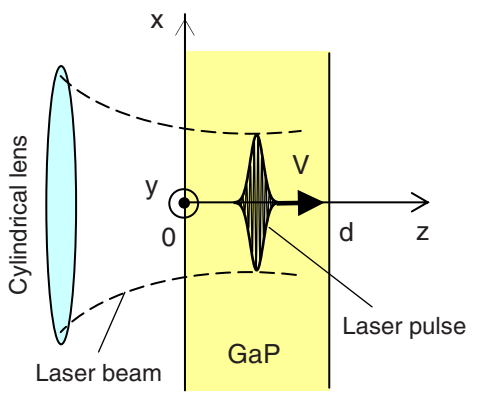

FIG. 2. (Color online) Schematics of the problem: a Ti:sapphire laser pulse focused in the $x$-direction by a cylindrical lens propagates through a slab of $\mathrm{GaP}$ with a group velocity $V=c / n_{g}$.

and spatial-spectral distribution of the terahertz emission from the slab. Practical applications to terahertz apertureless near-field microscopy are discussed.

\section{MODEL AND APPROACH}

Let us consider a slab $(0<z<d)$ of GaP illuminated normally by a Ti:sapphire laser pulse ( $800 \mathrm{~nm}$ wavelength) focused in the $x$-direction by a cylindrical lens (Fig. 2). The beam width in this direction $\ell_{\perp}$ can be varied in a wide range $\left(\ell_{\perp \mathrm{FWHM}}=2 \sqrt{\ln 2} \ell_{\perp}\right)$. In the $y$-direction, the beam width is assumed to be much greater than the terahertz wavelength. This allows us to approximate the laser by a 2D pulse with fields independent of $y$. The pulse propagates through the slab with a group velocity $V=c / n_{g}, n_{g}=3.56$ at $800 \mathrm{~nm}{ }^{9}$

We neglect the optical pulse distortion due to dispersion, diffraction, and pump depletion and write the nonlinear polarization induced in the slab via optical rectification as a given function of $x$ and $\xi=t-z / V$,

$$
\mathbf{P}^{\mathrm{NL}}=\mathbf{p} G(x) F(\xi) .
$$

In Eq. (1), the transverse profile of the optical beam $G(x)$ and the time-dependent envelope of the optical intensity $F(\xi)$ are assumed to be Gaussian functions

$$
G(x)=e^{-x^{2} / \ell_{\perp}^{2}}, F(\xi)=e^{-\xi^{2} / \tau^{2}},
$$

with the Fourier transforms

$$
\tilde{G}(g)=\frac{\ell_{\perp}}{2 \pi^{1 / 2}} e^{-g^{2} \ell_{\perp}^{2} / 4}, \quad \tilde{F}(\omega)=\frac{\tau}{2 \pi^{1 / 2}} e^{-\omega^{2} \tau^{2} / 4} .
$$

We assume the optimal for all zinc-blend crystals configuration of GaP (Ref. 4) - $\mathrm{a}\langle 110\rangle$-cut crystal with the [001] crystallographic axis oriented at an angle of $\approx 55^{\circ}$ with respect to the electric field of a linearly polarized, along the $x$-axis, laser beam. In this configuration, $\mathbf{p}$ is maximal and codirectional with the electric field; $p_{y}=p_{z}=0, p_{x}=d_{\mathrm{eff}} E_{0}^{2}$ with $d_{\mathrm{eff}}$ $=24.8 \mathrm{pm} / \mathrm{V}$ the nonlinear coefficient, ${ }^{12,13}$ and $E_{0}$ is the amplitude of the optical field in the crystal $\left[E_{0}\right.$ relates to the peak laser intensity $I_{0}$ as $I_{0}=(c / 8 \pi) n_{\text {opt }} E_{0}^{2}$, with $n_{\text {opt }}=3.18$ the refractive index of GaP at $800 \mathrm{~nm}$ (Ref. 12)]. The polarization $p_{x}$ excites $p$-polarized terahertz waves with field components $B_{y}, E_{x}$, and $E_{z}$. Excitation of $p$-polarized waves is advantageous (as compared to $s$-polarized waves with $E_{y}, B_{x}$, and $B_{z}$ ) due to higher transmission of these waves to vacuum at the exit boundary of the crystal. 
For the dielectric function of $\mathrm{GaP}$ in the terahertz range we use the one-phonon-resonance formula

$$
\varepsilon=\varepsilon_{\infty}+\frac{\left(\varepsilon_{0}-\varepsilon_{\infty}\right) \omega_{\mathrm{TO}}^{2}}{\omega_{\mathrm{TO}}^{2}-\omega^{2}+i \gamma \omega},
$$

with $\varepsilon_{0}=11.15, \varepsilon_{\infty}=9.35$, and $\omega_{\mathrm{TO}} /(2 \pi)=11.01 \mathrm{THz}{ }^{9}$ For the damping rate $\gamma$ we use a value $\gamma=4.6 \mathrm{THz}$ calculated from the experimental data of Ref. 14 on terahertz absorption in $\mathrm{GaP}$ at room temperature.

To find the terahertz radiation generated by the moving nonlinear polarization [Eq. (1)], we apply the general formulas of Ref. 4 to our case of $\mathbf{p}\left(p_{x}, 0,0\right)$. The magnetic field Fourier transform $\widetilde{B}_{y}(z, g, \omega)$ is given by

$$
\widetilde{B}_{y}= \begin{cases}D_{1} e^{i \kappa_{v} z}, & z<0 \\ D_{2} e^{-i \kappa_{c} z}+D_{3} e^{i \kappa_{c} z}-R e^{-i \omega z / V}, & 0<z<d \\ D_{4} e^{-i \kappa_{v}(z-d)}, & z>d,\end{cases}
$$

with

$$
\begin{aligned}
R= & 4 \pi p_{x} n_{g} \widetilde{F} \widetilde{G} /\left(c^{2} \kappa_{c}^{2} / \omega^{2}-n_{g}^{2}\right) \\
D_{1}= & D_{2}+D_{3}-R \\
D_{4}= & D_{2} e^{-i \kappa_{c} d}+D_{3} e^{i \kappa_{c} d}-R e^{-i \omega d / V} \\
D_{2,3}= & R\left[\left(\kappa_{c} \pm \varepsilon \kappa_{v}\right)\left(\varepsilon \kappa_{v}+\kappa_{c}^{2} V / \omega\right) e^{ \pm i \kappa_{c} d}+\left(\kappa_{c} \mp \varepsilon \kappa_{v}\right)\right. \\
& \left.\times\left(\varepsilon \kappa_{v}-\kappa_{c}^{2} V / \omega\right) e^{-i \omega d / V}\right] / \Lambda, \\
\Lambda= & \left(\kappa_{c}+\varepsilon \kappa_{v}\right)^{2} e^{i \kappa_{c} d}-\left(\kappa_{c}-\varepsilon \kappa_{v}\right)^{2} e^{-i \kappa_{c} d} .
\end{aligned}
$$

In Eqs. (5) and (6), the longitudinal wave numbers in vacuum $\kappa_{v}$ and in the crystal $\kappa_{c}$ are given by $\kappa_{v}^{2}=\omega^{2} / c^{2}$ $-g^{2}$ and $\kappa_{c}^{2}=\omega^{2} \varepsilon / c^{2}-g^{2}$. The Fourier transforms $\widetilde{E}_{x}(z, g, \omega)$ and $\widetilde{E}_{z}(z, g, \omega)$ can be expressed through $\widetilde{B}_{y}(z, g, \omega)$ by means of Maxwell's equations. ${ }^{4}$ To transform the solution (5) and (6) to the $t, x$ domain we take inverse transforms in the form

$$
B_{y}(z, x, t)=\int_{-\infty}^{\infty} d \omega \int_{-\infty}^{\infty} d g e^{i \omega t-i g x} \widetilde{B}_{y}(z, g, \omega)
$$

and the same formulas used for the other fields.

\section{RESULTS AND DISCUSSION}

\section{A. Emission from a planar optical pulse: Double inversion of terahertz pulses}

To get an analytical insight into the dynamics of terahertz generation in the strongly subluminal regime, let us consider first the limiting case of a planar optical pulse with $\ell_{\perp} \rightarrow \infty$. In this limit, the function $\widetilde{G}(g)$ in the coefficient $R$ transforms to the delta function $\delta(g)$ and the double Fourier integral [Eq. (7)] reduces to a simple integral. Additionally, let us set aside for a while the effects that arise from the presence of the exit boundary of the crystal at $z=d$ and consider first the crystal as semi-infinite $(d \rightarrow \infty)$. In practice (for $d>100 \mu \mathrm{m})$, the duration of the generated terahertz pulse is typically smaller than the roundtrip time of the pulse in the crystal and the processes at the entrance and exit boundaries of the crystal can be considered successively. ${ }^{15}$

Using $\ell_{\perp} \rightarrow \infty$ and $d \rightarrow \infty$, Eqs. (5), (6a)-(6e), and (7) for $z>0$ can be reduced to

$$
\begin{aligned}
E_{x}(z, t)= & 4 \pi p_{x} \int_{-\infty}^{\infty} d \omega \frac{\tilde{F}(\omega)}{\varepsilon(\omega)-n_{g}^{2}} \\
& \times\left[\frac{1+n_{g}}{1+\sqrt{\varepsilon(\omega)}} e^{i \omega(t-\sqrt{\varepsilon(\omega)} z / c)}-e^{i \omega(t-z / V)}\right],
\end{aligned}
$$

where, for convenience of practical application, electric field $E_{x}$ is used instead of $B_{y}$. In Eq. (8), according to the general concept of Ref. 4, the first term in the brackets is the freewave response that propagates with distortion due to dispersion and absorption and the second term is the forced-wave response that propagates without changing its shape.

In the case of a subluminal material $\left(n_{g}^{2}>\varepsilon_{0}\right)$, there is a peculiarity in the integrand of Eq. (8) at $\varepsilon(\omega)-n_{g}^{2}=0$. In the weakly subluminal regime of ZnTe excited with a Ti:sapphire laser, the peculiarity plays a significant role. The residue contributions from the poles $\varepsilon(\omega)-n_{g}^{2}=0$ describe the generation of a phase-matched wave. ${ }^{4}$ In the strongly subluminal regime of $\mathrm{GaP}$, the contributions from the poles are suppressed by the Gaussian factor $\widetilde{F}(\omega)$ (Fig. 1). Moreover, due to the flatness of the dispersion curve and small terahertz absorption in GaP (see Sec. I), we can approximate $\varepsilon(\omega)$ by a real constant $\varepsilon_{0}$. This allows us to evaluate the integral [Eq. (8)] analytically,

$$
E_{x}(z, t)=\frac{4 \pi p_{x}}{n_{0}^{2}-n_{g}^{2}}\left[\frac{1+n_{g}}{1+n_{0}} F\left(t-\frac{z n_{0}}{c}\right)-F\left(t-\frac{z n_{g}}{c}\right)\right]
$$

with $n_{0}=\sqrt{\varepsilon_{0}}$. Equation (9) was obtained earlier ${ }^{4}$ as a dispersionless approximation for the superluminal regimes of terahertz generation in $\mathrm{LiNbO}_{3}$ (excited at $\sim 800 \mathrm{~nm}$ ) and $\mathrm{GaAs}$ (excited at $\sim 1.5 \mu \mathrm{m}$ ). It predicts the generation of two terahertz pulses of the same (Gaussian) shape, which mimics the envelope of optical intensity but with different amplitudes and opposite signs. Physically, the free-wave pulse (first term in the brackets) is the transition radiation propagating from the entrance boundary of the crystal and the forced-wave pulse (second term in the brackets) is the near field of the nonlinear source. In contrast to superluminal materials (such as $\mathrm{LiNbO}_{3}$ and $\mathrm{GaAs}$ ), in $\mathrm{GaP}$ (excited at $\sim 800 \mathrm{~nm}$ ) the velocity $c / n_{0}$ of the free-wave pulse is higher than the velocity $V=c / n_{g}$ of the forced-wave pulse. Thus, in GaP the pulses propagate in inverse order as compared to superluminal materials - the free pulse ahead of the forced one. Furthermore, since the factor $n_{0}^{2}-n_{g}^{2}$ in Eq. (9) has opposite signs in $\mathrm{GaP}$ and in superluminal materials, the pulses in $\mathrm{GaP}$ have inverse polarities comparatively to $\mathrm{LiNbO}_{3}$ or GaAs. Thus, in $\mathrm{GaP}$ excited at $\sim 800 \mathrm{~nm}$ we have double (positionpolarity) inversion of the generated terahertz pulses with respect to the superluminal regime of terahertz generation, for example, in $\mathrm{LiNbO}_{3}$ or GaAs.

To illustrate the unusual dynamics of terahertz generation in the strongly subluminal regime, we plotted the snapshots of the electric field $E_{x}(z, t)$ in $\mathrm{GaP}$ on the basis of Eq. (8) with $\varepsilon(\omega)$ given by Eq. (4) (Fig. 3). Due to proximity of 


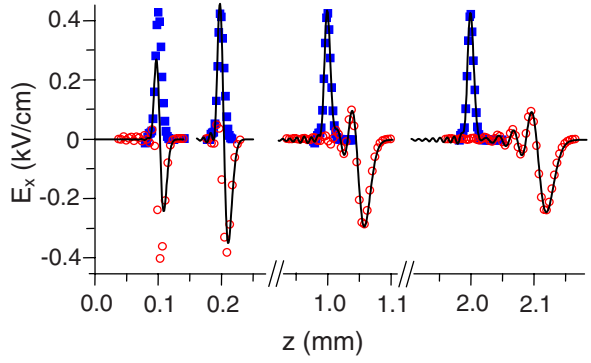

FIG. 3. (Color online) The free-(open circles) and forced-wave (filled squares) pulses and the total terahertz field $E_{x}(z, t)$ (solid line) in GaP plotted on the basis of Eq. (8) for successive moments of time. The laser pulse duration is $\tau_{\mathrm{FWHM}}=150$ fs and the peak laser intensity is $I_{0}=1 \mathrm{GW} / \mathrm{cm}^{2}$.

$n_{g}=3.56$ and $n_{0}=3.34$ the forced- and free-wave pulses have almost equal amplitudes [see Eq. (9)] and practically cancel each other near the entrance boundary $z=0$. The total terahertz field grows gradually with $z$ from zero and until the pulses become separated, i.e., within the walk-off length $L_{w}{ }^{4}$ To calculate $L_{w}$, we use Eq. (20) of Ref. 4 for the superluminal regime but with permuted terms $n_{g}$ and $n_{0}$, i.e., $L_{w}$ $=c \tau_{\mathrm{FWHM}} /\left(n_{g}-n_{0}\right)$. It gives $L_{w} \approx 200 \mu \mathrm{m}$ for $\tau_{\mathrm{FWHM}}$ $=150$ fs. For $0<z<L_{w}$, Eq. (9) can be reduced to

$$
E_{x} \approx-4 \pi p_{x} z\left[c\left(n_{g}+n_{0}\right)\right]^{-1} d F / d \xi
$$

which means that the total terahertz field is proportional to the derivative of the optical pulse intensity envelope and the passed distance $z$.

In Fig. 3, the snapshots at small distances $0<z \lesssim L_{w}$ look very similar to that for the weakly superluminal regime in GaAs (Ref. 4) [or in GaP pumped at $1.034 \mu \mathrm{m}$ (Ref. 16) _ - two adjacent pulses of opposite polarities, the negative pulse is ahead of the positive one. However, this similarity camouflages the double-inversion difference in the structure of the terahertz fields in the two regimes. This difference manifests itself at larger $z$. Indeed, in Fig. 3 for $z \gtrsim 1 \mathrm{~mm}$ the leading negative (free-wave) pulse experiences absorption and dispersive broadening while the delaying positive (forced-wave) pulse does not experience any change. On the contrary, in GaAs the delaying positive (free-wave) pulse experiences absorption and dispersive broadening while the leading negative (forced-wave) pulse does not change with $z^{4}$

To calculate the terahertz fields emitted to vacuum through the exit boundary $z=d$, one should apply the inverse Fourier transform [Eq. (7)] to the term $D_{4} e^{-i \kappa_{v}(z-d)}$ in Eq. (5) $\left(\right.$ at $\left.\ell_{\perp} \rightarrow \infty\right)$. A more simple approach is applying the transmission coefficients to the terahertz pulses [Eq. (9) or Eq. (8)]_usual Fresnel coefficient $T_{F}=2 n_{0} /\left(1+n_{0}\right)$ to the freewave pulse and new, introduced in Ref. 15, coefficient $T_{N}$ $=\left(n_{g}+n_{0}\right) /\left(1+n_{0}\right)$ to the forced-wave pulse. This approach, that omits multiple reflections of the terahertz fields from the slab's boundaries, is adequate to a practical situation where only the first (direct) terahertz pulse is typically used. Due to proximity of $n_{g}$ and $n_{0}$ the coefficients $T_{N}$ and $T_{F}$ are close, $T_{N} \approx 1.59, T_{F} \approx 1.54$, and, therefore, the ratio of the amplitudes of the forced- and free-wave pulses practically does not change after transmission through the boundary.
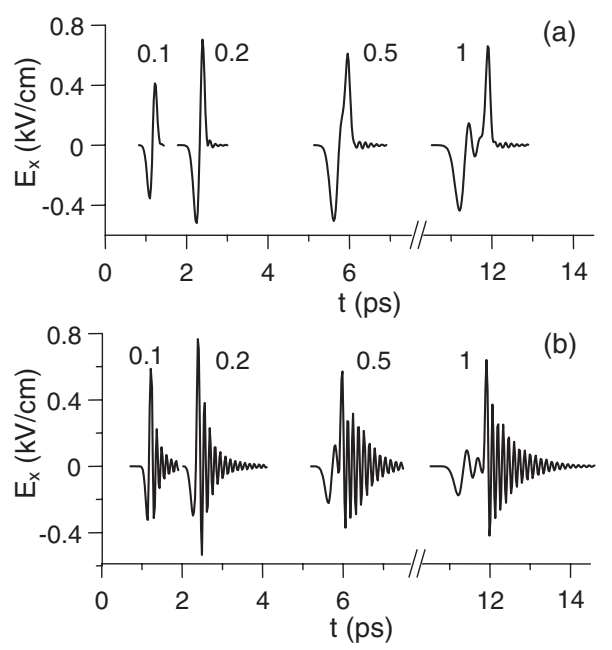

FIG. 4. Terahertz waveforms emitted from a slab of GaP of different thicknesses $d$ (shown in millimeter near the corresponding curves) for (a) $\tau_{\mathrm{FWHM}}=150$ fs and (b) $\tau_{\mathrm{FWHM}}=50$ fs. The peak laser intensity is $I_{0}$ $=1 \mathrm{GW} / \mathrm{cm}^{2}$.

Figure 4(a) shows the emitted from the crystal terahertz waveforms calculated using accurate formulas (5), (6a)-(6e), and (7) (at $\left.\ell_{\perp} \rightarrow \infty\right)$ for the same parameters as in Fig. 3. It is seen from Figs. 3 and 4(a) that the emitted from the crystal terahertz field is, indeed, $\sim 1.5-1.6$ times larger than in the crystal, the ratio of the amplitudes of the forced- and freewave pulses being practically the same both in vacuum and in the crystal.

From the practical point of view, the crystal thickness $d \approx 0.2-0.5 \mathrm{~mm}$ is optimal for the generation of a singlecycle high amplitude terahertz pulse, according to Fig. 4(a). Indeed, for $d<0.2 \mathrm{~mm}$ the amplitude of the terahertz pulse decreases, while increasing $d$ above $0.5 \mathrm{~mm}$ leads to generating a more complicated waveform and finally to splitting of the waveform into two separate pulses [Fig. 4(a)].

To demonstrate that shortening of the pump pulse duration can dramatically change the regime of terahertz generation from the strongly subluminal to weakly subluminal, we plotted the emitted from the slab terahertz waveforms for $\tau_{\text {FWHM }}=50$ fs [Fig. 4(b)]. A characteristic feature of Fig. 4(b) is the presence of an oscillating tail behind the forcedwave pulse. This tail is the phase-matched wave of the frequency $\omega_{0} \approx 7.4 \mathrm{THz}$. The build-up length $L_{b}$, introduced in Ref. 4 for characterizing stages of terahertz generation in the weakly subluminal regime, is estimated as $L_{b} \approx 200 \mu \mathrm{m}$ for GaP excited with Ti:sapphire laser. While $d \lesssim L_{b}$ the generated terahertz pulse grows in magnitude with $d$ without significant change in its shape [see oscillograms for 0.1 and 0.2 $\mathrm{mm}$ in Fig. 4(b)]. For $d>L_{b}$, the pulse grows in duration with $d$ without significant change in amplitude until the duration reaches the decay time of the phase-matched wave $\gamma / 2 \approx 400$ fs at $d \sim(0.5-1) \mathrm{mm}$. Increasing $d$ above $\sim(0.5-1) \mathrm{mm}$ does not influence the forced-wave response (i.e., the near field pulse and the phase-matched wave), while absorption and dispersive broadening of the leading freewave pulse become more pronounced for larger $d$ [see oscillograms for 0.5 and $1 \mathrm{~mm}$ in Fig. 4(b)]. The oscillograms in Fig. 4(b) agree perfectly with recent experimental results of Ref. 17. 

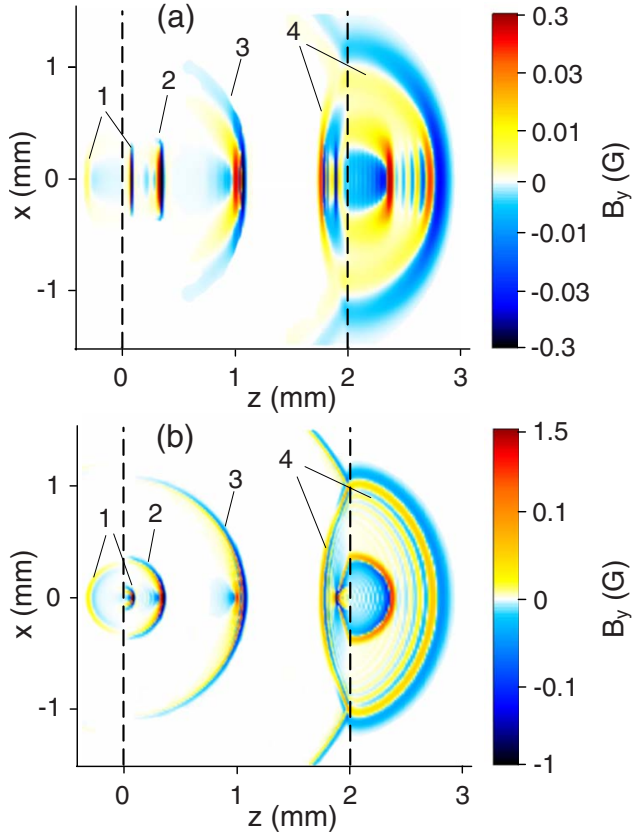

FIG. 5. (Color online) Snapshots of $B_{y}(z, x, t)$ at successive moments of time (numerated in order) for (a) $\ell_{\perp \mathrm{FWHM}}=300 \mu \mathrm{m}$ and (b) $\ell_{\perp \mathrm{FWHM}}$ $=30 \mu \mathrm{m}$. The laser pulse duration is $\tau_{\mathrm{FWHM}}=150 \mathrm{fs}$ and the peak laser intensity $I_{0}$ is (a) $0.1 \mathrm{GW} / \mathrm{cm}^{2}$ and (b) $1 \mathrm{GW} / \mathrm{cm}^{2}$. The crystal thickness is $d=2 \mathrm{~mm}$ and the crystal boundaries are shown by the dashed lines. In vacuum, a magnetic field of $1 \mathrm{G}$ corresponds to an electric field of 0.3 $\mathrm{kV} / \mathrm{cm}$.

\section{B. Emission from a focused optical pulse: Pointlike terahertz source}

Let us consider now the terahertz emission from optical pulses of an arbitrary transverse size. Figure 5 shows the spatial distribution of the magnetic field $B_{y}$ calculated on the basis of Eqs. (5), (6a)-(6e), and (7) for several successive moments of time and two values of the laser beam width $\ell_{\perp \mathrm{FWHM}}=300$ and $30 \mu \mathrm{m}$. In the calculations, the energy of the optical pulse (per unit length in the $y$-direction) was fixed to $0.5 \mu \mathrm{J} / \mathrm{cm}$, so the peak laser intensity $I_{0}$ differs by a factor of 10 in Figs. 5(a) and 5(b): 0.1 and $1 \mathrm{GW} / \mathrm{cm}^{2}$, respectively.

In the case of weak focusing [Fig. 5(a)], the generated terahertz field evolves inside the GaP crystal in accord with the one-dimensional (1D) theory of Sec. III A (moments $1-3)$ : the free- and forced-wave pulses practically cancel each other near the entrance boundary $z=0$ (moment 1 ); for $0<z \leqq 0.2 \mathrm{~mm}$ the total terahertz field behaves according to Eq. (10), i.e., it follows the derivative of the optical intensity envelope and grows with $z$ (moment 2); oscillations in the waveform (moment 3) arise due to dispersive distortion of the leading free-wave pulse. Incidence of the generated terahertz field on the exit boundary of the crystal produces the reflected and transmitted terahertz radiations (moment 4); the transmitted to the air magnetic field is reduced by a factor of $\sim 0.45-0.46$ due to reduction in the refractive index. One can see the formation of the far field in the leading part of the output radiation produced by the free-wave terahertz pulse (moment 4). The reflected transient radiation in the air $(z$ $<0)$ is barely discernible in Fig. 5(a) (moment 1) because of its weakness.

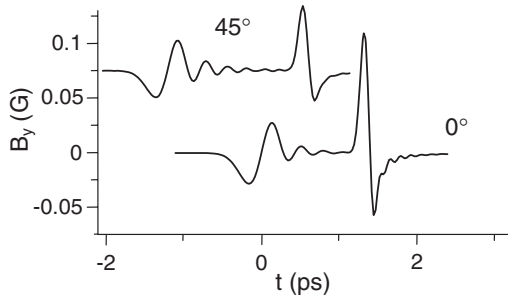

FIG. 6. Terahertz waveforms emitted from the slab of $\mathrm{GaP}$ at the angles of $0^{\circ}$ and $45^{\circ}$ (shown near the corresponding curves) to the $z$-axis after propagating a $2 \mathrm{~mm}$ distance in vacuum (in far field). Parameters are the same as in Fig. 5(b).

In the case of strong focusing [Fig. 5(b)], the radiation pattern produced by the laser pulse moving inside the crystal (moments 1-3) consists of a peak of the near field that accompanies the laser pulse, and a cylindrical wave propagating from the entrance boundary $z=0$ in the $+z$ direction ahead of the laser pulse (there is also weak transient radiation in the air propagating in the opposite direction, shown for moment 1). These two parts of the radiation pattern can be attributed, respectively, to the forced- $\left(-R e^{-i \omega z / V}\right)$ and freewave $\left(D_{2} e^{-i \kappa_{c} z}\right)$ terms in Eq. (5). The radiation pattern [Fig. 5(b)] differs significantly from the patterns typical for the superluminal and weakly subluminal regimes (Ref. 4) by the absence of a Cherenkov cone and phase-matched wave. Incidence of the cylindrical wave on the exit boundary of the crystal produces (moment 4 ) a relatively weak leading part in the output terahertz radiation (the pattern nulls in the reflected radiation correspond to the reflection at Brewster angle $\arctan n_{0}^{-1} \approx 17^{\circ}$ ). Incidence of the forced-wave pulse with a $d\left(n_{g}-n_{0}\right) / c \approx 1.5$ ps delay on the exit boundary gives $\sim 3$ times stronger single-cycle pulse in the output terahertz radiation (see oscillograms in Fig. 6).

For practical applications, the weak oscillating part of the output radiation can be filtered out using, for example, electro-optic sampling. Moreover, the delay and contrast in magnitude between the two parts of the output radiationthe leading oscillating part and the delayed single-cycle pulse - can be made more pronounced by using thicker crystals and focusing the pump laser pulse to a plane near the exit boundary of the crystal. ${ }^{18}$ In this case, the unfocused, at the entrance boundary of the crystal, laser pulse will excite the transient cylindrical wave only weakly and, additionally, the wave will experience a strong attenuation while propagating through a thick crystal. At the same time, the forcedwave pulse, excited by the focused laser pulse near the exit boundary of the crystal, will produce a strong single-cycle pulse in the output terahertz radiation.

An interesting peculiarity of the radiation pattern [Fig. 5 (b)] is that the single-cycle part of the output radiation has almost perfectly cylindrical wave front (the corresponding part of the reflected to the crystal radiation consists of both a cylindrical wave and two plane lateral waves). The magnitude of the terahertz field on the front only slightly decreases with the angle of emission counted from the $z$-axis (compare the oscillograms for two angles in Fig. 6). Such a radiation pattern can be explained by the emission of the single-cycle pulse from a narrow (with a width defined by the transverse 
size of the laser pulse) area on the exit surface of the crystal. Thus, in the strongly subluminal regime of optical-toterahertz conversion one can implement a threadlike-or pointlike, in the $3 \mathrm{D}$ case of focusing the laser beam to a spot-terahertz source that emits terahertz radiation almost isotropically. In the superluminal and weakly subluminal regimes, an implementation of such a source is impeded by the generation of the Cherenkov cone or phase-matched wave, respectively.

From the practical point of view, a possibility to reduce the size of the emitting area on the exit surface of the nonlinear crystal can be important, for example, for improving the resolution of the terahertz emission microscopy, ${ }^{19-21}$ a recently proposed type of terahertz apertureless near-field microscopy. In this technique, samples are deposited directly on the exit surface of the nonlinear crystal (ZnTe in Refs. 19-21) and illuminated by the generated in the crystal terahertz radiation in the near field mode. The resolution of the terahertz images obtained with the terahertz emission microscope is defined mainly by the size of the terahertz spot on the exit surface of the crystal and can be increased, in principle, by using the strongly subluminal regime of terahertz generation in $\mathrm{GaP}$.

In Ref. 19, electro-optic sampling was used to detect the terahertz radiation transmitted through the sample. Applying this technique to our scheme with GaP, the leading (oscillating) part of the terahertz radiation can easily be filtered out. If bolometer is used as a detector, like in Refs. 20 and 21, the response time of a bolometer (typically, in microsecond or even millisecond range) exceeds significantly the time delay between the two parts of terahertz radiation $(\sim 1 \mathrm{ps})$ and, thus, does not allow one to filter out the leading part of the radiation. Meanwhile, the emission of this part of the radiation is distributed along the exit surface over an area whose size depends on the crystal thickness and can exceed significantly the width of the optical beam [Fig. 5(b)]. To reduce the contribution to the bolometer response from the nonlocalized leading part of the radiation, one can apply the proposed above method-focusing the laser pulse to a plane near the exit boundary of a thick crystal (in this case $\ell_{\perp}$ decreases with $z$ inside the crystal). Moreover, even in the simple geometry that we consider in this paper, i.e., for $\ell_{\perp}$ independent of $z$ (Sec. II), using a GaP emitter instead of ZnTe of Refs. 19-21 can increase the resolution of the terahertz emission microscope. To demonstrate that, let us calculate the distribution of the total terahertz fluence, i.e., the total terahertz energy emitted per unit area of the exit surface, along the $x$-axis.

The terahertz fluence can be found by integrating the $z$-component of the Poynting vector $S_{z}$ in vacuum (at $z=d+$ ) over infinite interval of time $(-\infty<t<\infty)$. To find $S_{z}$ $=c(4 \pi)^{-1} E_{x} B_{y}$, we use the relation between the Fourier components of the electric and magnetic fields in vacuum $\widetilde{E}_{x}$ $=\left(c \kappa_{v} / \omega\right) \tilde{B}_{y}$ and the inverse transform of $\tilde{E}_{x}$ calculated according to Eq. (7). On integrating $S_{z}$, we obtain the fluence
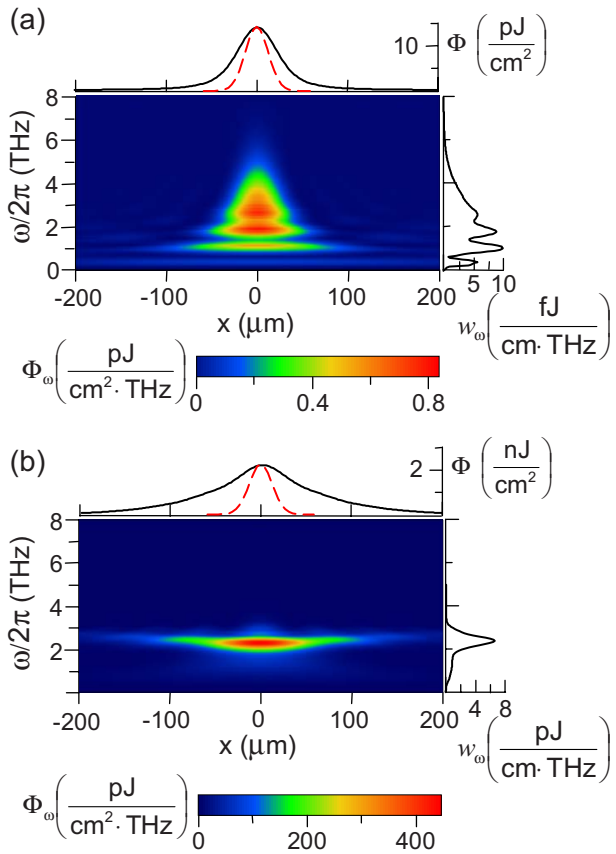

FIG. 7. (Color online) The distributions $\Phi_{\omega}(\omega, x), \Phi(x)$, and $w_{\omega}(\omega)$ for (a) $\mathrm{GaP}$ and (b) ZnTe crystals excited with Ti:sapphire laser $\left(\tau_{\mathrm{FWHM}}\right.$ $\left.=150 \mathrm{fs}, \ell_{\perp \mathrm{FWHM}}=30 \mu \mathrm{m}, I_{0}=1 \mathrm{GW} / \mathrm{cm}^{2}\right)$. The crystal thickness is $2 \mathrm{~mm}$. The dashed curves show the normalized profile of optical intensity.

$$
\Phi(x)=\int_{0}^{\infty} d \omega \Phi_{\omega}(\omega, x),
$$

where the spectral density of fluence is

$$
\begin{aligned}
\Phi_{\omega}(\omega, x)= & c \int_{-\omega / c}^{\omega / c} d g \int_{-\omega / c}^{\omega / c} d g^{\prime} e^{-i\left(g+g^{\prime}\right) x} \\
& \times \sqrt{1-c^{2} g^{2} / \omega^{2}} \operatorname{Re}\left[D_{4}(\omega, g) D_{4}\left(-\omega, g^{\prime}\right)\right]
\end{aligned}
$$

(Re denotes taking the real part). In Eq. (12), only propagating (with $|g|,\left|g^{\prime}\right|<\omega / c$ ) waves are included. To filter out the terahertz pulses that experience double and multiple reflections from the crystal's boundaries, we reduce Eq. (6c) to

$$
D_{4}=\frac{\varepsilon \kappa_{v}+\kappa_{c}^{2} V / \omega}{\varepsilon \kappa_{v}+\kappa_{c}} T_{F} R e^{-i \kappa_{c} d}-T_{N} R e^{-i \omega d / V},
$$

with $T_{F}$ and $T_{N}$ the transmission coefficients for the obliquely incident on the crystal-air boundary Fourier components of the free- and forced-wave pulses, respectively,

$$
T_{F}=\frac{2 \kappa_{c}}{\kappa_{c}+\varepsilon \kappa_{v}}, \quad T_{N}=\frac{\kappa_{c}\left(1+\kappa_{c} V / \omega\right)}{\kappa_{c}+\varepsilon \kappa_{v}} .
$$

Equation (14) for $T_{N}$ generalizes the result of Ref. 15 to the case of oblique incidence and $T_{F}$ is usual Fresnel coefficient.

Figure 7 shows the distributions $\Phi_{\omega}(\omega, x)$ (2D graphs) and $\Phi(x)$ (1D graphs at the top of each panel) calculated on the basis of Eqs. (11)-(14) for $2 \mathrm{~mm}$ slabs of GaP and ZnTe. For ZnTe, we use four-phonon-resonance dielectric function ${ }^{4}$ and $d_{\text {eff }}=68.5 \mathrm{pm} / \mathrm{V} .{ }^{12}$ Figure 7 shows also the spectral density of emitted terahertz energy $w_{\omega}(\omega)=\int_{-\infty}^{\infty} d x \Phi_{\omega}(\omega, x)$ (1D graphs at the right hand side of each panel). The distribution $\Phi(x)$ is twice as narrow for GaP [Fig. 7(a)] than for ZnTe [Fig. 7(b)] - the curve widths (FWHM) are $\approx 54$ and 


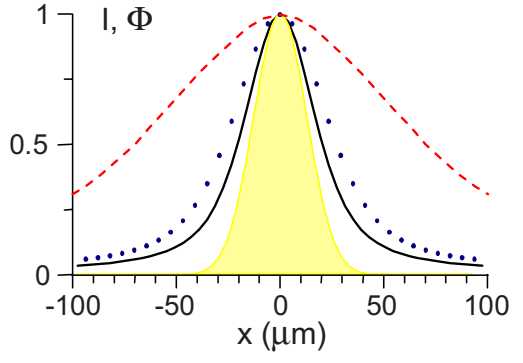

FIG. 8. (Color online) The normalized distributions $I(x)$ for GaP (solid) and ZnTe (dashed) crystals of a $2 \mathrm{~mm}$ thickness excited with Ti:sapphire laser $\left(\tau_{\mathrm{FWHM}}=150 \mathrm{fs}, \ell_{\perp \mathrm{FWHM}}=30 \mu \mathrm{m}\right)$. The dotted curve is $\Phi(x)$ for GaP. The shaded area shows the normalized profile of optical intensity.

$\approx 110 \mu \mathrm{m}$, respectively. In the case of $\mathrm{ZnTe}$, the terahertz energy comes from the phase-matched wave with frequency of $\approx 2.5 \mathrm{THz}$ [Fig. 7(b)]. The terahertz emission from GaP has a wide spectrum modulated with a period that is defined by the time delay between the two parts of terahertz radiation [Fig. 7(a)].

The width of the fluence distribution $\Phi(x)$ is an adequate characteristic of the resolution only for samples thicker than the terahertz wavelength. Indeed, Eq. (12) does not include evanescent modes with imaginary $\kappa_{v}$, i.e., with $1-c^{2} g^{2} / \omega^{2}$ $<0$. Meanwhile, a thin sample (with a thickness smaller than the terahertz wavelength) is illuminated by both the propagating $\left(1-c^{2} g^{2} / \omega^{2}>0\right)$ and evanescent $\left(1-c^{2} g^{2} / \omega^{2}<0\right)$ waves. For the case of thin samples, we plotted (Fig. 8) $x$-distribution of the integrated terahertz intensity on the exit boundary (at $z=d+$ ),

$$
I(x)=\int_{-\infty}^{\infty} d t B_{y}^{2}(z=d+, x, t)
$$

for $\mathrm{GaP}$ and $\mathrm{ZnTe}$. According to Fig. 8, the distribution $I(x)$ for $\mathrm{GaP}$ is 1.25 times more narrow than $\Phi(x)$ for this crystal and 3.3 times more narrow than $I(x)$ for ZnTe. Interestingly, the width of $I(x)$ for ZnTe $(142 \mu \mathrm{m})$ is larger than the width of $\Phi(x)$ for this crystal $(110 \mu \mathrm{m})$.

The difference in the width of $I(x)$ between $\mathrm{GaP}$ and ZnTe becomes less striking for thinner ZnTe crystals. For example, for $200 \mu \mathrm{m}$ thick ZnTe used in experiment ${ }^{21}$ the width equals $89 \mu \mathrm{m}$. It is smaller than for $2 \mathrm{~mm}$ thick ZnTe (142 $\mu \mathrm{m}$ ) but still more than two times as large as for $2 \mathrm{~mm}$ thick GaP $(43 \mu \mathrm{m})$. To obtain the width of $I(x)$ comparable to GaP, the thickness of ZnTe crystal should be as small as $50 \mu \mathrm{m}$. Even though the magnitude of the terahertz intensity for $50 \mu \mathrm{m}$ thick ZnTe is $\sim 4$ times as high as for $2 \mathrm{~mm}$ thick $\mathrm{GaP}$, so thin $(50 \mu \mathrm{m})$ crystals are inconvenient for practical use.

\section{Fundamental limitations on the size of the output terahertz spot}

To realize the potentials of improving the terahertz emission microscopy resolution, let us address now a general question: are there any limitations on the minimal size of the output terahertz spot even for an infinitely sharp $\left(\ell_{\perp} \rightarrow 0\right)$ focusing of the pump laser pulse? In principle, such limitations can come from two physical factors-a restriction from

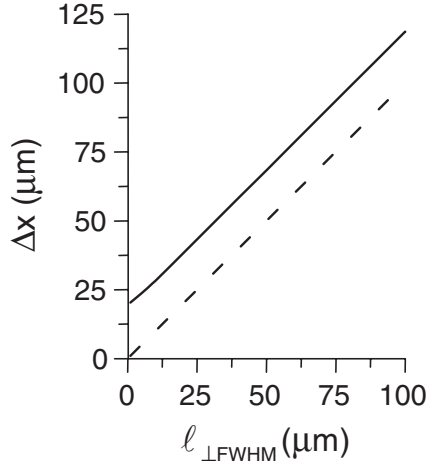

FIG. 9. The transverse size of the terahertz near field $\Delta x$ (at $\xi=0$ ) as a function of $\ell_{\perp \text { FWHM }}$ plotted on the basis of Eq. (7) for $\tau_{\mathrm{FWHM}}=150 \mathrm{fs}$ (solid). The dashed straight line $\Delta x=\ell_{\perp \text { FWHM }}$ is shown for reference.

below on the transverse size of the forced-wave (near-field) pulse inside the crystal and broadening of the terahertz intensity distribution due to inhomogeneous transmission of the terahertz spatial harmonics (with different $g$ ) through the exit boundary of the crystal.

To estimate the minimal size of the near-field inside the crystal, we substitute $\widetilde{B}_{y}=-R e^{-i \omega z / V}$ to the integral [Eq. (7)] and evaluate this integral analytically in the dispersionless approximation $\left[\varepsilon(\omega) \approx n_{0}^{2}\right]$. For a strongly focused laser pulse with $\ell_{\perp} \ll c \tau\left(n_{g}^{2}-n_{0}^{2}\right)^{-1 / 2}$, Eq. (7) can be reduced to the following explicit form:

$$
B_{y}(x, \xi)=\frac{4 \pi n_{g} p_{x} \ell_{\perp}}{c \tau \sqrt{n_{g}^{2}-n_{0}^{2}}} \operatorname{Re}\left[1-\sqrt{\pi} u e^{u^{2}} \operatorname{erfc}(u)\right],
$$

where $\operatorname{erfc}(u)$ is the complementary error function and $u$ $=|x|\left(n_{g}^{2}-n_{0}^{2}\right)^{1 / 2} /(c \tau)-i \xi / \tau$. Since $\ell_{\perp}$ does not enter parameter $u$, the spatial distribution of the near field produced by a strongly focused [with $\ell_{\perp} \ll c \tau\left(n_{g}^{2}-n_{0}^{2}\right)^{-1 / 2}$ ] laser pulse is independent of $\ell_{\perp}$. The magnitude of the field does not depend on $\ell_{\perp}$ as well for a fixed power of the laser pulse, $p_{x} \ell_{\perp}$ =const. Thus, the width (FWHM) of the distribution [Eq. (16)] along the $x$-axis at $\xi=0$ (at midpulse) gives the minimal possible size of the near field

$$
(\Delta x)_{\min } \approx 0.87 c \tau\left(n_{g}^{2}-n_{0}^{2}\right)^{-1 / 2} .
$$

Interestingly, according to Eq. (17) $(\Delta x)_{\min }$ is proportional to the laser pulse duration $\tau$. For example, for $\tau_{\mathrm{FWHM}}=150 \mathrm{fs}$ we obtain $(\Delta x)_{\min } \approx 19 \mu \mathrm{m}$ in $\mathrm{GaP}$. The minimal width $(\delta x)_{\min }$ of the intensity distribution $B_{y}^{2}(x, \xi=0)$ is more than twice as small,

$$
(\delta x)_{\min } \approx 0.41 c \tau\left(n_{g}^{2}-n_{0}^{2}\right)^{-1 / 2},
$$

i.e., $(\delta x)_{\min } \approx 9 \mu \mathrm{m}$ for $\tau_{\mathrm{FWHM}}=150$ fs. Figure 9, plotted on the basis of accurate Eq. (7) with $\widetilde{B}_{y}=-R e^{-i \omega z / V}$, shows that the transverse size (at $\xi=0$ ) of the near field $\Delta x$, indeed, tends to $(\Delta x)_{\min }$ given by Eq. (17), in the limit $\ell_{\perp} \rightarrow 0$. Interestingly, the difference between the transverse sizes of the near field and the laser pulse is $\approx(\Delta x)_{\text {min }}$ in a wide interval of $\ell_{\perp}$ (two lines in Fig. 9 are practically parallel).

Figure 10 shows the limiting (at $\ell_{\perp} \rightarrow 0$ ) form of the near field, calculated on the basis of Eq. (7) with $\widetilde{B}_{y}=$ $-R e^{-i \omega z / V}$ and $\ell_{\perp} \rightarrow 0$ in $R\left(p_{x} \ell_{\perp}=\right.$ const for a fixed energy of 


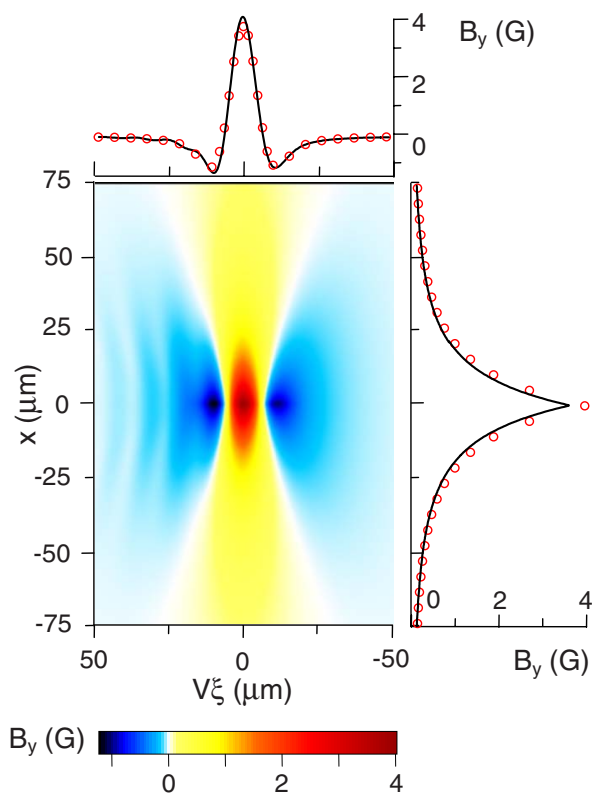

FIG. 10. (Color online) The limiting (at $\ell_{\perp} \rightarrow 0$ ) spatial distribution of the near field $B_{y}(x, \xi)$ calculated on the basis of Eq. (7) for $\tau_{\mathrm{FWHM}}=150 \mathrm{fs}$ and the laser pulse energy of $0.5 \mu \mathrm{J} / \mathrm{cm}$. The transverse (at $\xi=0$ ) and longitudinal (at $x=0$ ) profiles of the field are plotted on the basis of Eq. (7) (solid line) and Eq. (16) (open circles).

the laser pulse). (Note that the $z$ and $V \xi$ axes have opposite directions.) The results of the calculations agree perfectly with the dispersionless approximation [Eq. (16)] (Fig. 10). The field decays as $\propto x^{-2}$ in the lateral directions. In contrast to the planar pulse approximation (Sec. III A), the longitudinal profile of the near field in Fig. 10 does not mimic the envelope of the optical intensity. The near field has a shorter duration $(\approx 100 \mathrm{fs}, \mathrm{FWHM})$ and a more complicated shape than the laser pulse.

Addressing the broadening of the terahertz field due to transmission through the exit boundary of the crystal, we plotted the limiting (at $\ell_{\perp} \rightarrow 0$ ) integrated intensities $I^{ \pm}(x)$ on the opposite sides of the boundary-at $z=d \pm$, respectively (Fig. 11). In the calculations, we used Eqs. (7) and (15) with $\widetilde{B}_{y}=-R e^{-i \omega d / V}$, for $z=d-$, and $\widetilde{B}_{y}=-T_{N} R e^{-i \omega d / V}$, for $z=d+$, and $\ell_{\perp} \rightarrow 0$ in $R\left(p_{x} \ell_{\perp}=\right.$ const $)$. It is seen from Fig. 11 that transmission through the boundary, indeed, significantly-by a factor of $\sim 1.5$ - broadens the terahertz intensity distribution, thus, increasing its minimal width from $\approx 10 \mu \mathrm{m}$ at $z$ $=d-$ to $\approx 16 \mu \mathrm{m}$ at $z=d+$. This effect can be explained by a different phase change experienced by terahertz spatial harmonics (with different $g$ ) when they are transmitted through the boundary.

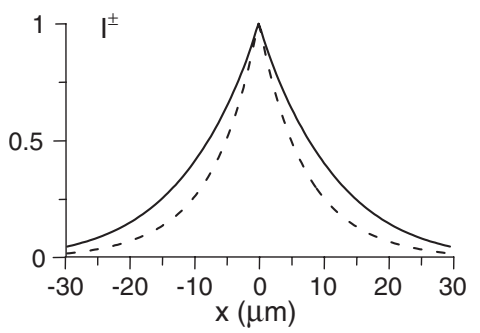

FIG. 11. The normalized limiting (at $\ell_{\perp} \rightarrow 0$ ) distributions $I^{ \pm}(x)$ (solid and dashed, respectively) on the opposite sides of the exit boundary of GaP for $\tau_{\mathrm{FWHM}}=150 \mathrm{fs}$.
Thus, the minimal possible size of the output terahertz spot at the exit boundary of a GaP crystal excited with a Ti:sapphire laser is $\approx 16 \mu \mathrm{m}$ for $\tau_{\mathrm{FWHM}}=150 \mathrm{fs}$. For a further decrease in the spot size, shorter laser pulses should be used but that would inevitably lead to a shift in the generated terahertz spectrum to higher frequencies-the peak spectral frequency of the field (16) (at $x=0)$ is $\omega_{\text {peak }}=\sqrt{2} \tau^{-1}$.

\section{CONCLUSION}

To conclude, we have introduced and investigated the strongly subluminal regime of terahertz generation with ultrashort laser pulses in electro-optic crystals. This regime is achieved when the group velocity of the pump laser pulse is smaller than the highest phase velocity of terahertz waves in the crystal $\left(n_{g}>n_{0}\right)$ and, additionally, the spectral bandwidth of the laser pulse is smaller than the frequency of the phasematched terahertz wave $\left(2 \tau^{-1} \ll \omega_{0}\right)$. These conditions can be fulfilled, for example, for $\mathrm{GaP}$ excited with Ti:sapphire laser pulses of typical duration $\left(\tau_{\mathrm{FWHM}} \gtrsim 100 \mathrm{fs}\right)$.

We have studied the peculiarities of the terahertz emission from a slab of GaP excited with a Ti:sapphire laser. For a weakly focused laser pulse, the emitted from the crystal terahertz pulses demonstrate double (position-polarity) inversion as compared to the emission from $\mathrm{LiNbO}_{3}$ or GaAs. The optimal thickness of GaP crystal for generating single-cycle high amplitude terahertz pulses is $0.2-0.5 \mathrm{~nm}$.

For a strongly focused laser pulse, the strongly subluminal regime offers a practically interesting possibility to reduce the size of the terahertz spot at the exit surface of the crystal [by a factor of $\sim(2-3)$ for GaP with respect to $\mathrm{ZnTe}$ ]. This can be used for increasing the resolution of terahertz apertureless near-field microscopy.

Finally, we have found fundamental limitations on the minimal size of the terahertz spot on the exit boundary of the crystal that follow from a restriction from below on the transverse size of the terahertz near-field of the nonlinear source and from the spatial broadening of the terahertz field when it is transmitted through the crystal boundary.

\section{ACKNOWLEDGMENTS}

We are grateful to Dr. A. V. Maslov for fruitful discussions. This work was supported in part by RF President Grant No. MK-3749.2008.2 and RFBR Grant Nos. 08-0200988, 08-02-92216, and 08-02-99052. M.I.B. and M.T. acknowledge partial support from the joint research program "Studies of nonlinear optical processes for efficient terahertz generation," funded by Research Center for Development of Far-Infrared Region, University of Fukui. S.B.B. acknowledges partial support from the fund "Dynasty."

${ }^{1}$ T. E. Stevens, J. K. Wahlstrand, J. Kuhl, and R. Merlin, Science 291, 627 (2001).

${ }^{2}$ J. K. Wahlstrand and R. Merlin, Phys. Rev. B 68, 054301 (2003).

${ }^{3}$ G. A. Askar'yan, Zh. Eksp. Teor. Fiz. 42, 1360 (1962) [Sov. Phys. JETP 15, 943 (1962)]; Phys. Rev. Lett. 57, 2470 (1986).

${ }^{4}$ M. I. Bakunov, S. B. Bodrov, A. V. Maslov, and M. Hangyo, Phys. Rev. B 76, 085346 (2007).

${ }^{5}$ A. Nahata, A. S. Weling, and T. F. Heinz, Appl. Phys. Lett. 69, 2321 (1996).

${ }^{6}$ T. Loffler, T. Hahn, M. Thomson, F. Jacob, and H. G. Roskos, Opt. Ex- 
press 13, 5353 (2005).

7F. Blanchard, L. Razzari, H.-C. Bandulet, G. Sharma, R. Morandotti, J.-C. Kieffer, T. Ozaki, M. Reid, H. F. Tiedje, H. K. Haugen, and F. A. Hegmann, Opt. Express 15, 13212 (2007).

${ }^{8}$ G. Chang, C. J. Divin, C.-H. Liu, S. L. Williamson, A. Galvanauskas, and T. B. Norris, Opt. Express 14, 7909 (2006).

${ }^{9}$ E. D. Palik, Handbook of Optical Constants of Solids (Academic, Orlando, 1985).

${ }^{10}$ A. G. Stepanov, J. Hebling, and J. Kuhl, Appl. Phys. B: Lasers Opt. 81, 23 (2005).

${ }^{11}$ A. G. Stepanov, J. Kuhl, I. Z. Kozma, E. Riedle, G. Almasi, and J. Hebling, Opt. Express 13, 5762 (2005)

${ }^{12}$ J. Hebling, A. G. Stepanov, G. Almási, B. Bartal, and J. Kuhl, Appl. Phys. B: Lasers Opt. 78, 593 (2004).

${ }^{13} \mathrm{All}$ formulas in the paper are in cgs units. To convert the nonlinear coef- ficient $d_{\text {eff }}$ given in SI to cgs one should use following rule: $d[\mathrm{~cm} / \mathrm{CGSE}]=\left(3 \cdot 10^{-8} / 4 \pi\right) d[\mathrm{pm} / \mathrm{V}]$.

${ }^{14}$ T. Tanabe, K. Suto, J. Nishizawa, K. Saito, and T. Kimura, Appl. Phys. Lett. 83, 237 (2003).

${ }^{15}$ M. I. Bakunov, A. V. Maslov, and S. B. Bodrov, Phys. Rev. Lett. 99, 203904 (2007).

${ }^{16}$ Y. J. Ding, IEEE J. Sel. Top. Quantum Electron. 10, 1171 (2004).

${ }^{17}$ R. Chakkittakandy, J. A. W. M. Corver, and P. C. M. Planken, Opt. Express 16, 12794 (2008).

${ }^{18}$ W. Yan, Y.-P. Yang, H. Chen, and L. Wang, Phys. Rev. B 75, 085323 (2007).

${ }^{19}$ K. Wynne and D. A. Jaroszynski, Opt. Lett. 24, 25 (1999).

${ }^{20}$ R. Lecaque, S. Gréssilon, N. Barbey, R. Peretti, J.-C. Rivoal, and C. Boccara, Opt. Commun. 262, 125 (2006).

${ }^{21}$ R. Lecaque, S. Gréssilon, and C. Boccara, Opt. Express 16, 4731 (2008). 